\title{
Nativity, Neighborhoods, and Body Composition in the Sacramento Area Latino Study on Aging
}

\author{
Eva M. Durazo, PhD, ${ }^{1,2}$ Mary N. Haan, DrPH, ${ }^{1, *, 0}$ Kristina Dang, MPH, ${ }^{1}$ Allison E. Aiello, \\ $\mathrm{PhD}{ }^{3}$ and Jacqueline M. Torres, $\mathrm{PhD}^{1}$
}

'Department of Epidemiology and Biostatistics, University of California, San Francisco, California. ${ }^{2}$ Center for the Study of Adversity and Cardiovascular Disease (NURTURE Center), Division of Cardiology, Department of Medicine, University of California, San Francisco, California. ${ }^{3}$ Department of Epidemiology, University of North Carolina at Chapel Hill, Gillings School of Global Public Health, Chapel Hill, North Carolina.

*Address correspondence to: Mary N. Haan, DrPH, Department of Epidemiology and Biostatistics, University of California, 550 16th Street, San Francisco, CA 94158. E-mail: Mary.Haan@ucsf.edu

Received: April 30, 2019; Editorial Decision Date: September 19, 2019

Decision Editor: Suzanne Meeks, PhD

\begin{abstract}
Background and Objectives: Globally, obesity influences the risk of many major chronic diseases. Our study examines the association between individual nativity and neighborhood level concentration of immigrants with 10-year changes in weight, body mass index (BMI), and waist circumference (WC) among older Latinos.

Research Design and Methods: The Sacramento Area Latino Study on Aging (SALSA) is a population-based prospective study of community-dwelling older adults of Mexican origin (baseline ages 58-101 years). The primary outcome was repeated measures of weight over a 10-year period for 1,628 respondents. Nativity was defined by participants' reported place of birth (US-born or Latin American foreign born). Neighborhood immigrant concentration was measured as the percentage of foreign born at census tract level (2000 US Census). We used linear mixed models with repeated measures of weight, height, BMI, and WC as dependent variables (level 1), clustered within individuals (level 2) and neighborhood migrant concentration (level 3).

Results: Foreign born (FB) respondents had lower baseline weight than the US-born (mean, 160 vs. $171 \mathrm{lbs}, p<.0001$ ). Over time, weight differences between the FB and the US-born decreased by $1.7 \mathrm{lbs} / 5$ years as US-born weight decreased more rapidly. We observed a significant interaction between individual nativity and neighborhood immigrant concentration $(p=.012)$. We found similar patterns for BMI, but did not find statistically significant differences in WC trajectories.

Discussion and Implications: Our study observed significant differences by foreign born vs. US nativity in baseline weight/ BMI and in their trajectories over time. Additionally, we found weight/BMI differences in neighborhood immigrant concentration for the FB, but not for the US-born.
\end{abstract}

Keywords: Weight, BMI, Latinos/Hispanic

Obesity and overweight are important risk factors for major chronic diseases (Cetin \& Nasr, 2014; Jensen et al., 2014; Jiang et al., 2018; Mathus-Vliegen, 2012). In the United States, over a third of the population is obese, and prevalence is higher among specific race/ethnic subgroups, such as African Americans and Latinos (Ogden, Carroll, Fryar, \& Flegal, 2015; Wang, Colditz, \& Kuntz, 2007). Occurrence of obesity is related to age: Fakhouri and colleagues reported that obesity may be higher in those $65-74$ years of age $(40.8 \%$,$) , compared with those aged 75$ and over 
(27.8\%) (Fakhouri, Ogden, Carroll, Kit, \& Flegal, 2012). Some research has shown that obesity in older adults is a risk factor for several diseases, disability, and even mortality (Cetin \& Nasr, 2014; Jackson, 2015; Jiang et al., 2018; Mathus-Vliegen, 2012). Several studies have reported that midlife obesity is associated with dementia while late life obesity is not (Lavie, McAuley, Church, Milani, \& Blair, 2014; Whitmer, Gunderson, Barrett-Connor, Quesenberry, \& Yaffe, 2005; Whitmer et al., 2008).

In aging populations, change in obesity and weight are complex factors; although obesity can be a risk factor for other diseases/outcomes, weight and body composition (i.e., fat distribution, muscle mass, and height) are often found to decrease with increasing age (Allen, Clarke, Shipley, \& Leon, 2019; Cetin \& Nasr, 2014; Miller \& Wolfe, 2008; Rothman, 2008). A large number of studies in older populations have reported that weight loss is associated with increased risk of death (Park et al., 2018). Both obesity and weight loss can be risk factors of poor health in older age.

In the United States, an average of $39 \%$ of all Latinos aged 20-84 are obese (Isasi et al., 2015). The prevalence of obesity has been shown to differ by ethnicity and nativity (US-born or foreign born). US-born Latinos on average have a higher body mass index (BMI) than the foreign born (FB). Some research suggests the longer the foreign born live in the United States, the higher their weight and BMI (Albrecht, Diez Roux, Aiello, Schulz, \& AbraidoLanza, 2013; Albrecht, Roux, et al., 2013; Bates, AcevedoGarcia, Alegría, \& Krieger, 2008; Isasi et al., 2015). The differences by nativity observed in obesity and body mass index among Latinos may be explained by the healthy immigrant theory (Escarce, Morales, \& Rumbaut, 2006; Markides \& Rote, 2019). This advantage is theorized to occur due to migration by healthier individuals and/or healthier behaviors of immigrants, such as diet and higher levels of physical activity (Markides \& Rote, 2019; Turra \& Elo, 2008). However, less is known about nativity and change in body composition patterns over time, particularly in older ages.

Individual nativity may influence preferential selection of residence and health behaviors. In turn, neighborhood concentration of migrants may also affect health behaviors which could affect body composition and weight changes over time. The upstream influence of nativity or neighborhood on weight change has not been addressed, although nativity or neighborhood appears to be associated with several other health outcomes, including mortality (AfableMunsuz, Mayeda, Pérez-Stable, \& Haan, 2014). Figure 1 presents our conceptual framework for this study where we focus on the association of nativity with change in weight over time. We examined the potential modifying effect of neighborhood immigrant concentration, based on healthrelated selection and ethnic enclave theories. We identified several potential-mediating factors in our framework, but they are not included as covariates in the final model

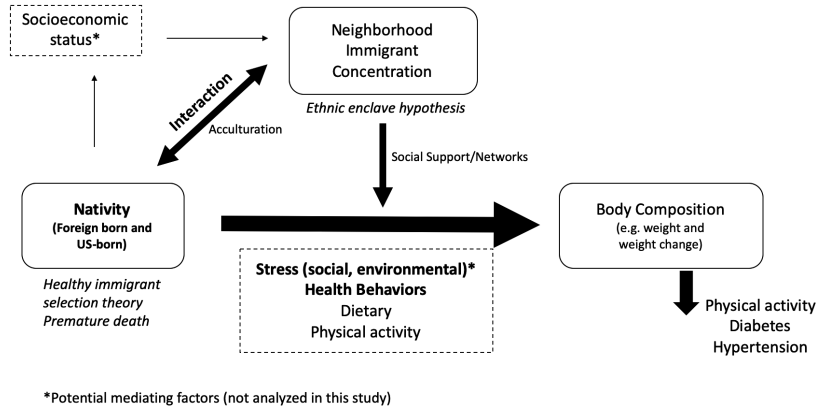

Figure 1. Theoretical framework of nativity and weight association, moderated by immigrant neighborhood concentration.

(Model 1 in Table 2) to minimize unnecessary adjustment in the analysis (Schisterman, Cole, \& Platt, 2009).

Analyses of the health impact of neighborhood features have produced mixed findings within Latino populations. Some studies suggest that ethnic enclaves (i.e., defined here as high percent Latino or immigrant neighborhoods) might be protective for the health of Latino residents (Eschbach, Ostir, Patel, Markides, \& Goodwin, 2004; Markides \& Rote, 2019; Yen, Michael, \& Perdue, 2009). More recent findings challenge this notion, with benefits observed for some behaviors (e.g., diet low in fats and access to healthy foods), but negative associations observed for other outcomes (e.g., lower physical activity and higher cardiovascular mortality) (Durazo, Mbassa, \& Albert, 2016; Osypuk, Diez Roux, Hadley, \& Kandula, 2009; Rodriguez et al., 2018; Viruell-Fuentes, Morenoff, Williams, \& House, 2013). Other research has shown that patterns of body composition vary by individual nativity for Latinos; less is understood about how neighborhood immigration concentration might affect patterns of change in weight over time in individuals; few studies have examined whether individual nativity and neighborhood migrant concentration may interact to modify weight changes over time (Albrecht et al., 2015).

Neighborhood features may affect weight change in old age through several pathways in the physical and social environment. For example, the physical environment can influence physical activity resources available, and the social environment can provide the social network or support to mitigate stressors, which can influence weight (Diez Roux \& Mair, 2010). Cultural factors may be important in promoting healthy behaviors (López, Peralta, Lee, Zeki Al Hazzouri, \& Haan, 2014; Whitley et al., 2018). Having a neighborhood with high concentrations of immigrants might influence maintenance of dietary norms that result in healthier body composition, for example, because ethnic food options are more available in the immigrant enclave (Moore \& Diez Roux, 2006; Osypuk et al., 2009); this advantage may be restricted to those who favor those foods. Immigrant enclaves may offer resident Latinos more social cohesion and larger social networks (Portes \& Manning, 1986; Viruell-Fuentes et al., 2013). Greater social cohesion 
Table 1. Baseline Characteristics by Nativity

\begin{tabular}{|c|c|c|c|}
\hline \multirow[b]{4}{*}{ Variables } & \multicolumn{2}{|l|}{ Nativity } & \multirow[b]{4}{*}{$P$} \\
\hline & \multirow{3}{*}{$\begin{array}{l}\text { US Born } \\
(n=812) \\
\% \text { or Mean (SD) }\end{array}$} & \multirow{3}{*}{$\frac{\text { Foreign Born }}{(n=816)}$} & \\
\hline & & & \\
\hline & & & \\
\hline Age (years) & $69.93(6.26)$ & $71.04(7.61)$ & 0.0013 \\
\hline Gender & & & 0.111 \\
\hline Male & $43.60 \%$ & $39.71 \%$ & \\
\hline Female & $56.40 \%$ & $60.29 \%$ & \\
\hline Education (years) & $9.75(4.90)$ & $5.06(4.75)$ & $<0.0001$ \\
\hline Gross monthly household income & & & $<0.0001$ \\
\hline$<\mathrm{US} \$ 1000$ & $28.73 \%$ & $58.97 \%$ & \\
\hline US\$1000-US\$1499 & $19.15 \%$ & $21.08 \%$ & \\
\hline US\$1500-US\$1999 & $14.05 \%$ & $8.91 \%$ & \\
\hline US\$2000-US\$2499 & $14.05 \%$ & $5.90 \%$ & \\
\hline$\geq \mathrm{US} \$ 2500$ & $24.00 \%$ & $5.14 \%$ & \\
\hline Physical activity (MET hr/week) & $72.95(75.74)$ & $70.73(73.25)$ & 0.56 \\
\hline Physical activity & & & 0.41 \\
\hline High ( $\geq 35 \mathrm{MET}$ hr/week) & $63.31 \%$ & $61.26 \%$ & \\
\hline Low & $36.69 \%$ & $38.74 \%$ & \\
\hline Smoking & & & 0.03 \\
\hline Current & $11.10 \%$ & $11.78 \%$ & \\
\hline Former & $46.24 \%$ & $39.88 \%$ & \\
\hline Never & $42.66 \%$ & $48.34 \%$ & \\
\hline Depressive symptoms & & & $<0.0001$ \\
\hline Depression risk (CESD $\geq 16$ ) & $20.10 \%$ & $30.91 \%$ & \\
\hline No depression risk & $79.90 \%$ & $69.09 \%$ & \\
\hline Diabetes & & & $<0.0001$ \\
\hline Yes & $37.68 \%$ & $29.17 \%$ & \\
\hline No & $62.32 \%$ & $70.83 \%$ & \\
\hline Systolic blood pressure (mmHg) & $137.95(19.51)$ & $138.92(19.40)$ & 0.31 \\
\hline Diastolic blood pressure (mmHg) & $75.99(10.62)$ & $75.80(10.58)$ & 0.73 \\
\hline Hypertension & & & 0.201 \\
\hline Yes & $70.81 \%$ & $67.89 \%$ & \\
\hline No & $29.19 \%$ & $32.11 \%$ & \\
\hline Neighborhood percent immigrant & & & $<0.0001$ \\
\hline Low $(0-27.5 \%)$ & $40.39 \%$ & $27.08 \%$ & \\
\hline Medium ( $>27.5 \%$ to $50 \%)$ & $33.37 \%$ & $34.80 \%$ & \\
\hline $\operatorname{High}(>50 \%)$ & $26.23 \%$ & $38.11 \%$ & \\
\hline Weight (lbs) & $171.08(36.12)$ & $160.44(30.46)$ & $<0.0001$ \\
\hline Height (inches) & $63.30(4.22)$ & $62.18(3.84)$ & 0.008 \\
\hline BMI $\left(\mathrm{kg} / \mathrm{m}^{2}\right)$ & $30.10(5.91)$ & $29.28(5.34)$ & 0.003 \\
\hline BMI categories & & & 0.07 \\
\hline Obese $\left(\geq 30 \mathrm{~kg} / \mathrm{m}^{2}\right)$ & $44.70 \%$ & $39.83 \%$ & \\
\hline Overweight ( 25 to $<30 \mathrm{~kg} / \mathrm{m}^{2}$ ) & $38.05 \%$ & $39.22 \%$ & \\
\hline Normal/Underweight $\left(<25 \mathrm{~kg} / \mathrm{m}^{2}\right)$ & $17.24 \%$ & $20.96 \%$ & \\
\hline Waist circumference (inches) & $38.32(5.40)$ & $37.94(5.00)$ & 0.14 \\
\hline Waist circumference & & & 0.89 \\
\hline High (male $>40$ in; female $>35$ in) & $51.61 \%$ & $51.17 \%$ & \\
\hline Low (male $\leq 39$ in; female $\leq 34$ in) & $48.39 \%$ & $48.83 \%$ & \\
\hline
\end{tabular}

Sacramento Area Latino Study on Aging, $N=1,628$.

*Dropped cases missing baseline BMI. 
Table 2. Results From Multilevel Linear Regression Models of the Associations Between Nativity and Weight (Ibs) OverTime, Sacramento Area Latino Study on Aging. $N=1,628$

\begin{tabular}{|c|c|c|}
\hline & Model 1 & Model 2 \\
\hline Variable & $b(S E)$ & $b(S E)$ \\
\hline \multicolumn{3}{|l|}{ Nativity } \\
\hline US-born & Ref & Ref \\
\hline Foreign Born & $-7.31(1.45)^{* * * *}$ & $-11.04(2.61) * * *$ \\
\hline Time since baseline (years) & $-1.03(0.07)^{* * * *}$ & $-1.08(0.11) * * *$ \\
\hline \multicolumn{3}{|l|}{ Nativity*Time Interaction } \\
\hline US-Born*Time & Ref & Ref \\
\hline FB*Time & $0.34(0.11) * *$ & $0.40(0.18) *$ \\
\hline Height since baseline & $0.82(0.10)^{* * *}$ & $0.83(0.10) * * *$ \\
\hline Age (years) & $-1.23(0.10)^{* * * *}$ & $-1.21(0.10)^{* * *}$ \\
\hline \multicolumn{3}{|l|}{ Gender } \\
\hline Male & Ref & Ref \\
\hline Female & $-18.43(1.53) * * *$ & $-18.32(1.53) * * *$ \\
\hline Education (Years) & & $-0.01(0.15)$ \\
\hline \multicolumn{3}{|l|}{ Neighborhood (\% Immigrant) } \\
\hline Low immigrant $(0-27.5 \%)$ & & Ref \\
\hline Medium immigrant (>27.5\% to $50 \%$ ) & & $2.21(2.40)$ \\
\hline High immigrant $(>50 \%)$ & & $-3.01(2.62)$ \\
\hline \multicolumn{3}{|l|}{ Nativity*Neighborhood (\% Immigrant) } \\
\hline FB x Low Immigrant & & Ref \\
\hline FB x Medium Immigrant & & $2.22(3.53)$ \\
\hline FB $x$ High Immigrant & & $8.52(3.62) *$ \\
\hline \multicolumn{3}{|l|}{ Neighborhood (\% Immigrant)*Time } \\
\hline Low immigrant & & Ref \\
\hline Medium immigrant & & $0.12(0.17)$ \\
\hline High immigrant & & $0.06(0.19)$ \\
\hline \multicolumn{3}{|c|}{ Nativity*Neighborhood (\% Immigrant)*Time } \\
\hline FB $x$ Low Immigrant & & Ref \\
\hline FB x Medium Immigrant & & $-0.01(0.26)$ \\
\hline FB $x$ High Immigrant & & $-0.17(0.27)$ \\
\hline Intercept & $215.47(10.11) * * *$ & $213.86(10.54) * *$ \\
\hline AIC & 47343.55 & 47344.78 \\
\hline
\end{tabular}

$* p<.05 ; * * p<.01 ; * * p<.001$.

All covariates collected at baseline, except for time and height.

has been associated with less smoking and depression and more physical activity (Echeverría, Diez-Roux, Shea, Borrell, \& Jackson, 2008; Murillo, Echeverria, \& Vasquez, 2016); these are factors which can directly affect body composition. Neighborhoods with higher levels of social cohesion may mitigate the negative effects of other factors, such as low socioeconomic status, on overall health (Cramm, van Dijk, \& Nieboer, 2013). More research is needed to better understand the impact of neighborhoods for residents as they age. There may be important differences in late-life body composition outcomes by multi-level factors that reflect both individual and contextual dimensions related to immigration experience. By considering the context of an aging Latino population, such as the interactions between healthy immigrant selection theory and ethnic enclave research, we may better target and improve intervention efforts to ensure a healthy older population.
The aim of this study is to examine whether individual nativity and neighborhood-level immigrant concentration are associated with change over time in body weight, BMI, and waist circumference (WC) over a 10 -year period in a cohort of older Latinos. We further tested whether neighborhood immigrant concentration may modify the association between individual nativity and our outcomes. Based on previous research, we hypothesized that (a) foreign born Latinos would have lower baseline weight, BMI, and WC than US-born Latinos and (b) weight, BMI, and WC would decline over time for both FB and US-born Latinos (considering that weight declines with increasing age). We expected (c) neighborhood immigrant percentage to modify the association between individual nativity and body composition outcomes. Specifically, we expected that living in a neighborhood with higher concentrations of immigrants would result in individuals with less obesity at baseline 
and less change in weight (or BMI/WC), thus contributing to lower baseline body composition measures and slower declines over time than those living in low immigrant neighborhoods. We expected the higher percent immigrant neighborhood, to be protective of obesity for all of its residents regardless of nativity.

\section{Methods}

\section{Study Population}

The Sacramento Area Latino Study on Aging (SALSA) cohort is a longitudinal study of 1,789 community-dwelling older adults (aged 58-101 years) of Latin American-origin. About 95\% were of Mexican origin. Baseline data were collected in 1998-1999. Follow-up data collections continued every 12-15 months in participants' homes, for a total of six follow-ups to 2008. Brief phone interviews were done every 6 months. The study target area consisted of a 6-county area in the Sacramento Valley (Sacramento, Yolo, Yuba, Solano, Sutter, and Placer). 1998 US census tracts within these counties were selected if the Hispanic population was at least $5 \%$. Enumeration and recruitment were done by door to door canvasing in these tracts. Any household resident in 1998 or 1999 who self-identified as Hispanic, was age 60 or older, and was willing to participate was enrolled in the study. The response rate was $85 \%$ of contacted eligible people. The annual attrition rate was $5 \%$ overall, including deaths and loss to follow-up. The study design and participant characteristics have been described in greater detail (Haan et al., 2003). The current analysis excluded 161 participants with missing baseline BMI data, leaving a baseline analysis sample of 1,628. SALSA was approved by the institutional review boards of the University of Michigan and the Universities of California, San Francisco and Davis and University of North Carolina.

\section{Measures \\ Individual level measures}

\section{Anthropometric measures.}

In annual home visits, weight, height, and waist circumference were collected in person. Body mass index was calculated based on measured weight $(\mathrm{kg})$ divided by measured height $(\mathrm{m})$ squared $\left(\mathrm{kg} / \mathrm{m}^{2}\right)$. There is ongoing debate over the most accurate body composition measurements for older adults (Cetin \& Nasr, 2014; Rothman, 2008). Waist circumference was measured with a tape according to standard procedures. Since BMI is a ratio measure, over time it reflects both changes in weight and changes in height. One alternative method is to use weight as a main effect term and to adjust for height. Waist circumference has also been identified as a measure of central body fat and a predictor of cardiometabolic disease risk (Klein et al., 2007). Thus, we explored change in several body composition measures, including weight (height adjusted), BMI, and WC.

Individual nativity was self-reported at enrollment as country of birth and categorized in this analysis as US-born and FB. The foreign-born group consists of participants from Mexico (94\%) and Central America or South America (6\%).

Additional covariates, based on our previous cross-sectional analyses and other literature, included age (years), gender, and education (years completed).

\section{Neighborhood level measure}

Participant baseline addresses were geocoded and linked to the 2000 US Census tracts; this allowed us to merge the data with tract-level Census data (Zeki Al Hazzouri et al., 2011). Our neighborhood measure is operationalized using the concentration (i.e., percentage) of foreign born from Mexico or Central America in a census tract (mean of $41 \%$ ). There are many categories in the Census measuring immigration within a tract (e.g., year of migration and sending country). We chose to use the proportion of immigrants of Mexican or Central American origin as our neighborhood measure because it best reflected the ethnic composition of our participants and was comparable to other studies examining the neighborhood concept of immigrant enclaves (Li, Wen, \& Henry, 2017; Osypuk et al., 2009). A higher percentage indicated a higher concentration of immigrants. We categorized the percent immigrant tract variable into tertiles based on the sample distribution to distinguish between neighborhoods with low $(\leq 27.5 \%)$, medium ( $>27.5 \%$ to $50 \%$ ), and high ( $>50 \%$ ) percent immigrant.

\section{Statistical analyses}

Baseline characteristics are described as percentages or means in Table 1 . We tested for differences by nativity using chi-square tests for categorical variables or one-way analysis of variance for continuous variables. We used hierarchical linear mixed models to estimate associations between individual nativity and continuous outcomes of weight, BMI, or WC. Change was modeled over a 10 -year period of time with unbalanced correlated data. We used three levels of data: six time points for 1,628 individuals nested within 259 neighborhoods (i.e., census tracts). Among our sample, the size of the tract-specific population ranged from 1 to 192. The linear mixed models included subject-specific random intercept and subject-specific random slopes that allowed for varying trajectories of weight, BMI, or WC across individuals and were specified with an unstructured correlation matrix.

Figure 1 displays the pathways occupied by mediating or confounding covariates; Table 2 , Model 1, includes only nativity, baseline age, time and gender, and height; nativity is the main predictor and age; gender and height are treated as confounders (Schisterman et al., 2009). Model 2 tests for effect modification by adding neighborhood 
immigrant concentration (neighborhood), two-way interactions between Nativity*Neighborhood and Neighborhood*time, and a three-way interaction between Nativity*Neighborhood*Time. Model 2 is compared with Model 1 by AIC for the overall fit of the models. Mediators (health behaviors, diet, physical activity, acculturation, social support, diabetes, and hypertension) are not included in Table 2 analysis.

In a sensitivity analyses, we utilized a similar procedure, multilevel mixed-effects logistic regression, to estimate the effects of nativity and neighborhood on the odds of obesity $\left(\mathrm{BMI} \geq 30 \mathrm{~kg} / \mathrm{m}^{2}\right.$ ) over time. We wanted to better understand the associations to obesity, which could be a risk factor for other diseases. We also adjusted for additional covariates at baseline, specifically demographic, behavioral, and clinical risk factors (household income, physical activity level [measured in Metabolic Equivalents (METs)], smoking status, depressive symptoms [20-item version of the Center for Epidemiological Studies Depression scale, range 0-60], diabetes [determined by self-report physician diagnosis, use of diabetes medication, and/or fasting glucose level of $126 \mathrm{mg} / \mathrm{dL}$ or higher], and hypertension [self-reported physician diagnosis, use of hypertension medication, and/or a systolic blood pressure greater than $140 \mathrm{mmHg}$ or a diastolic blood pressure greater than $90 \mathrm{mmHg}]$ ) (Association, 2018; Carey \& Whelton, 2018; González, Haan, \& Hinton, 2001; HSPH, 2019). Model 3 in Supplementary Tables 1-3 includes the adjustment with all covariates. All analyses were performed in STATA version 15 .

\section{Results}

We present baseline characteristics by individual nativity in Table 1. Approximately half of the sample was foreign born (FB). FB individuals were older and had lower education and income levels compared with the US-born at baseline of the study. FB respondents were also less likely to have ever smoked, reported higher depressive symptoms, and had lower diabetes prevalence compared to US-born Latinos. There was no statistically significant difference in gender, physical activity, mean blood pressure, and hypertension by nativity. Socioeconomic factors at the individual and neighborhood levels may be associated with immigrant concentration; in this analysis, education and income were significantly lower in the FB vs. US-born (Table 1). Over forty-one percent of the US-born lived in low migrant tracts and $38 \%$ of $\mathrm{FB}$ lived in high migrant neighborhoods. At baseline, those in high and medium migrant neighborhoods weighed less $(5.89$ and $7.1 \mathrm{~kg})$ than those in low migrant areas.

At baseline, the US-born had higher values for all body composition measures; US-born Latinos weighed 11 pounds more than $\mathrm{FB}(p=.0001)$ and were 1.4 inches taller $(p=.008)$. Although small in magnitude, the mean difference $\left(0.8 \mathrm{~kg} / \mathrm{m}^{2}\right)$ in BMI by nativity was statistically significant $(p=.003)$, with the US-born having higher average BMI. (For example, an individual with height of $5^{\prime} 3^{\prime \prime}$ would need to lose approximately $5 \mathrm{lbs}$ to have a decrease in BMI of $.8 \mathrm{~kg} / \mathrm{m}^{2}$.) The US-born were also more likely to be obese and had larger WC measures than the FB. However, mean WC differences by nativity were not statistically significant.

Weight and BMI declined over time. Figure 2 shows the predicted marginal weight over time by individual nativity. Overall, weight declined by about a pound a year $(b=-0.4 \mathrm{~kg}, p<.001)$, BMI declined by $0.1 \mathrm{~kg} / \mathrm{m}^{2}(p<$ .001) per year, whereas waist circumference declined by less than a thousandth of an inch each year and was not statistically significant (data not shown). Baseline weight was higher and weight loss change was more rapid among U.S. born compared with foreign born (Figure 2).

Figure 3 shows the predicted marginal weight over time stratified by nativity by neighborhood concentration (tertiles). The slope of weight change was steeper and more divergent in Figure 3B than 3A. These are derived from Table 2, Model 2.

Table 2 presents the association between nativity and change in weight over time from a series of hierarchical linear mixed models. Model 1 included time, a nativity-time interaction, and was adjusted for height over time, baseline age, and gender. Initial weight was significantly lower in FB Latinos compared to US-born Latinos $(b=-7.3 \mathrm{lbs}, p$ $<.001)$. The nativity by time interaction was statistically significant, indicating a difference by nativity in the rate of change of weight over time $(b=0.3 \mathrm{lbs}$ in FB vs. US-born, $p<.01)$. Accordingly, the slope of weight change was less steep for the FB compared with the US-born (Figure 2). Although weight declined for both groups, over a 5-year period the difference in weight between the $\mathrm{FB}$ and the US-born decreased by nearly two pounds. (Change in weight estimate over 5 years: $(-7.31 \mathrm{lbs}+(.34 \mathrm{lbs} / \mathrm{yrs} * 5$ yrs $))=-5.61$. A difference of $(-7.31--5.61)=1.71 \mathrm{bs}$.

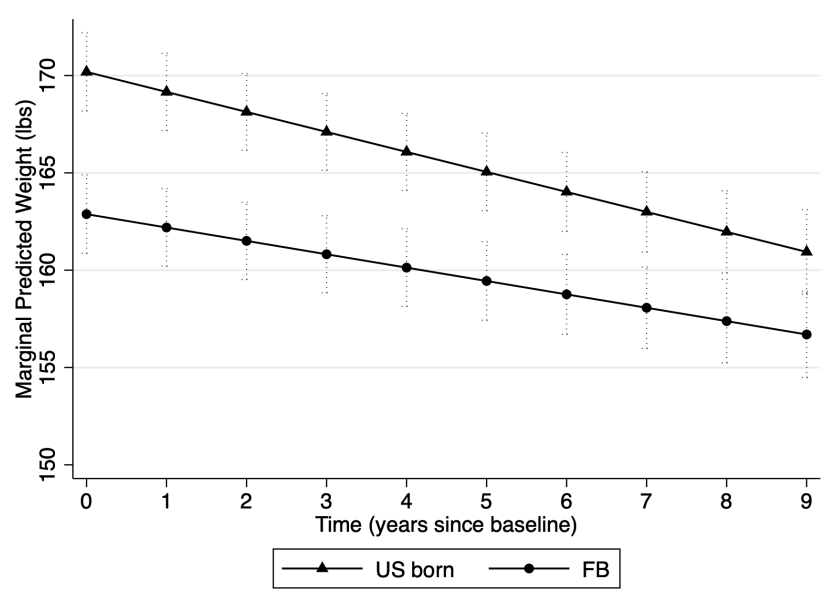

Figure 2. Predicted marginal weight by nativity, adjusted for age, gender, and height. 

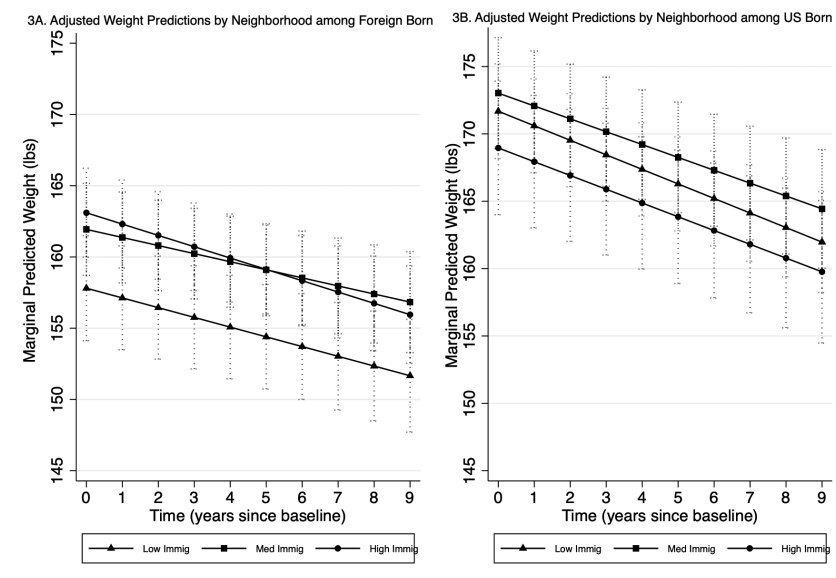

Figure 3. Predicted marginal weight by neighborhood type (\% immigrant) among the foreign born and US-born, fully adjusted model.

To examine the possible moderating effect of the neighborhood on the association between individual-level nativity and body composition outcomes, we included an interaction term between nativity and neighborhood immigrant concentration in Model 2. The nativityneighborhood interaction was statistically significant $(p=.02)$. FB respondents who lived in high immigrant neighborhoods had higher baseline weight than FB living in low immigrant neighborhoods $(b=8.5 \mathrm{lbs}, p<.05)$, with a difference by neighborhood continuing over time (see marginal predicted weight for FB by immigrant neighborhood concentration in Figure 3A). There were no significant differences in weight by neighborhood immigrant concentration among US-born Latinos, see Figure 3B. The two-way interaction of neighborhood-time and the threeway interaction of nativity-neighborhood-time were not statistically significant.

In Supplementary Tables, we present a similar series of linear mixed models with BMI (Supplementary Table 1S) and WC (Supplementary Table 2S) over time as the dependent variables. The association between nativity, neighborhood immigrant concentration, and BMI mirrored the patterns of weight. We did not find a significant association between nativity, neighborhood immigrant concentration, and WC. We also include Supplementary Table 3S, which includes Model 3, that adjusts for all demographic, behavioral, and clinical risk factors.

\section{Sensitivity Analysis}

We examined the odds of obesity (BMI $\geq 30 \mathrm{~kg} / \mathrm{m}^{2}$ ) over time using a mixed hierarchical model (STATA 15 melogit). After initially adjusting for time and baseline age, both time and baseline age were associated with lower odds of obesity [OR: 0.96 (95\% CI: $0.93-0.99, p=.01$ ) and OR: 0.85 $(0.82-0.89, p<.0001)]$, respectively. We next examined the odds of obesity by nativity, adjusting for time, baseline age, gender, and neighborhood percent immigrant. FB nativity was associated with lower odds of obesity compared to US-born, OR: 0.50 (95\% CI: 0.32-0.76, $p=.001$ ). Compared with those residing in the lowest tertile of neighborhood immigrant concentration, there were higher odds of obesity in the medium tertile, OR: 2.47 (95\% CI: 1.48$4.13, p=.001)$ and high tertile, OR: $3.32(1.95-5.62, p<$ .0001) immigrant percentage tracts. Furthermore, nativity and neighborhood exhibited an interaction such that odds of obesity in the FB who lived in a medium, OR: $4.21(95 \%$ CI: $1.50-11.81, p<.01)$ and high, OR: 7.58 (2.63-21.9, $p<.0001)$ immigrant neighborhood was higher compared with FB living in a low immigrant neighborhood.

These results reflect a declining risk of obesity in the study as a whole; these risks are modified by individual nativity and migrant concentration (census based) neighborhood such that FB Latinos compared with US born had a lower risk of obesity. FB who lived in a medium and high immigrant neighborhood had a higher risk of obesity at baseline compared with those in a low immigrant neighborhood. These associations for odds of obesity are similar to our findings for weight. In Supplementary Analysis (Supplementary Figure 1S), we observed the association between weight change by baseline obesity and nativity. Baseline obesity had an important influence on weight change over time such that predicted weight change over time was highest among baseline obese US-born compared both to all FB and to nonobese US-born.

\section{Discussion}

We examined the role of individual nativity and neighborhood-level nativity (immigrant concentration) on weight, BMI, and WC over time in a cohort of older Latinos. We found that, among older Latinos, weight and BMI decreased over time; this has been documented in other aging populations (Allen et al., 2019; Karahalios, English, \& Simpson, 2016). Foreign born Latinos had lower baseline weight and BMI than US-born Latinos, even with adjustment for age, gender, and height. Over time, the slopes of change for weight and BMI began at a higher level and declined more steeply for US-born compared with foreign born Latinos. It is likely that declining weight in old age reflects worsening health. Other authors have reported similar findings in samples of older people (Karahalios et al., 2016; Zajacova \& Ailshire, 2014). The faster decline in weight and BMI for US-born Latinos compared with FB may reflect differences in health status between the two groups. The implications of weight loss for health in older age have been debated-in part-because of the contravening possible health benefits of intentional weight loss for obese older adults (Shea et al., 2011; Witham \& Avenell, 2010). The more rapid decrease in weight and BMI in the US-born could result in some positive health outcomes, such as improved hypertension and glycemic control. However, there are notable negative health implications of weight loss in older ages; unintentional weight loss is related to 
physiologic markers of aging, including increased muscle loss, frailty, disability, cognitive decline, risk of dementia, and mortality (Ali \& Garcia, 2014; Bell et al., 2017; Cova et al., 2016; Gaddey \& Holder, 2014; Miller \& Wolfe, 2008). Compared with FB, US-born in our study experienced accelerated decline in weight and BMI, suggesting that the FB may be initially healthier and that health of the US-born declines more rapidly.

It is not completely clear what influences the differences in weight change between FB and US-born Latinos. Selective survival may lead US-born to die at a younger age because of their higher prevalence of obesity at baseline, coupled with more rapid decline in weight over time. US-born Latinos have lived more time in the US compared with FB Latinos, which could result in greater exposure to racial/ethnic discrimination, greater relative economic deprivation, or similar stressors which may negatively affect health over time (Pérez, Fortuna, \& Alegría, 2008); this may help account for the greater decline in weight for the US-born over time.

Our results suggest that immigrant neighborhood concentration may moderate the association between nativity and initial weight in the FB. The FB living in low immigrant neighborhoods had the lowest odds of obesity, with higher obesity odds for those in medium and high immigrant neighborhoods. In our sample, low migrant concentration tracts differed from high migrant tracts with respect to socioeconomic factors such as level of higher education, lower percent in poverty, and higher concentrations of more recent migrants. These findings suggest that possible health benefits for FB may be larger in neighborhoods with lower concentrations of migrants, which may also reflect the greater socioeconomic and social resources in those neighborhoods. FB Latinos living in low immigrant concentration neighborhoods had higher education and income levels as well as higher neighborhood socioeconomic status, all of which have been associated with better BMI and obesity outcomes. There may yet be unmeasured dimensions of socioeconomic status, such as wealth, associated with neighborhood immigrant concentration.

Our finding of differences in mean baseline weight for FB living in high immigrant neighborhoods compared with those living in low immigrant neighborhoods contradicts what has been found in ethnic enclave literature, which hypothesizes that high immigrant neighborhood concentrations are protective of health (i.e., mean lower baseline weight) (Ishizawa \& Jones, 2016; Li et al., 2017). We found the opposite to be true, with FB Latinos living in high immigrant neighborhoods having higher weight, BMI, and obesity risk. Similar to this finding, a study by Grafova and colleagues found that men over age 55 living in a high immigrant neighborhood were more likely to be obese (Grafova, Freedman, Kumar, \& Rogowski, 2008). There may be different neighborhood effects on body composition for certain subgroups, such as foreign-born older adults. There are no other studies of weight change by nativity or neighborhood migrant features among aging Latinos (McGrath, Vincent, Al Snih, Markides, \& Peterson, 2017; Park et al., 2018; Ullmann, Goldman, \& Pebley, 2013).

There are some limitations in our study that should be considered. The body composition measures available in this sample included weight, height, BMI, and waist circumference. Underlying age-related changes in muscle mass and fat mass and bone loss may not be precisely reflected in these measures, (Cetin \& Nasr, 2014; Han, Tajar, \& Lean, 2011; Rothman, 2008). Since there is debate as to the most useful measures and cut-points of body composition for older adults (Howel, 2012), we considered several measures. Our study has very limited information on dietary patterns and no information on intentional weight loss. Additionally, our sample consists primarily of Latinos of Mexican-origin living in the Sacramento Valley of California. Latinos from other countries of origin may have different patterns of body composition. Research from the Hispanic Community Health Study/Study of Latinos (HCHS/SOL) on obesity prevalence among middle-aged Latinos in the United States found some differences by ethnic subgroup; those of Puerto Rican descent had the highest rates of obesity and those of South American origin had the lowest (Isasi et al., 2015). They also found differences in obesity rates by location within the same ethnic groups, for example, obesity prevalence in Mexicanorigin respondents was higher among those from the Bronx compared with those from San Diego (Isasi et al., 2015).

Our study has many strengths. SALSA is a longitudinal cohort with body composition measures repeated annually over a 10-year period; this allowed us to examine weight change over time. Additionally, much of the previous research on BMI and WC trends are based on younger cohorts where weight is more likely to increase. Our study of body composition patterns among older Latinos provides additional understanding of how nativity status and neighborhood context can affect health risk factors in an aging population.

\section{Implications}

Our study sought to examine whether nativity of individuals and neighborhood-level nativity were associated with 10-year changes in body composition measures in an older Latino cohort. Our purpose was to identify whether individual and contextual migration may affect the risk of poorer health in older age. We found differences in weight and BMI by nativity and neighborhood at baseline and over time in our older sample. US-born Latinos had higher weight and BMI at baseline and a faster decline over time, compared with foreign born Latinos. Additionally, we observed differences by neighborhood immigrant concentration for foreign born Latinos at baseline, with those living in high immigrant neighborhoods 
having higher mean weight and BMI than those living in low immigrant neighborhoods. Our study suggests that compared with foreign born Latinos, US-born Latinos may experience deleterious changes in weight; this may be an important point of prevention and intervention in healthy aging. Additionally, foreign born living in high immigrant neighborhoods may not be benefiting from the postulated health benefits of living in an ethnic enclave. Future research should validate these nativity patterns in other disease outcomes, as well as with other immigrant populations and Latino subgroups. As the US aging population continues to grow, understanding differences among immigrant subgroups and neighborhood influence will be important as researchers and clinicians attempt to support a climate of healthy aging.

\section{Supplementary Material}

Supplementary data are available at The Gerontologist online.

\section{Funding}

E.M.D. is funded by a training grant through the National Institute of Aging T32-AG049663. This research is supported by the National Institutes of Health (NIH) grants AG12975 and DK60753 to M.N.H. The funders had no role in study design, data collection and analysis, decision to publish, or preparation of the manuscript.

\section{Conflict of Interest}

None reported.

\section{References}

Afable-Munsuz, A., Mayeda, E. R., Pérez-Stable, E. J., \& Haan, M. N. (2014). Immigrant generation and diabetes risk among Mexican Americans: the Sacramento area Latino study on aging. American Journal of Public Health, 104 Suppl 2, S234-S250. doi:10.2105/AJPH.2012.300969r

Albrecht, S. S., Diez Roux, A. V., Aiello, A. E., Schulz, A. J., \& Abraido-Lanza, A. F. (2013). Secular trends in the association between nativity/length of US residence with body mass index and waist circumference among Mexican-Americans, 19882008. International Journal of Public Health, 58, 573-581. doi:10.1007/s00038-012-0414-5

Albrecht, S. S., Osypuk, T. L., Kandula, N. R., Gallo, L. C., Lê-Scherban, F., Shrager, S., \& Diez Roux, A. V. (2015). Change in waist circumference with longer time in the United States among Hispanic and Chinese immigrants: the modifying role of the neighborhood built environment. Annals of Epidemiology, 25, 767-72.e2. doi:10.1016/j.annepidem.2015.07.003

Albrecht, S. S., Roux, A. V. D., Kandula, N. R., Osypuk, T. L., Ni, H., \& Shrager, S. (2013). Immigrant assimilation and BMI and waist size: a longitudinal examination among Hispanic and Chinese participants in the multi-ethnic study of atherosclerosis. Obesity (Silver Spring, Md.), 21, 1695-1703. doi:10.1002/ oby.20104
Ali, S., \& Garcia, J. M. (2014). Sarcopenia, cachexia and aging: diagnosis, mechanisms and therapeutic options - a mini-review. Gerontology, 60, 294-305. doi:10.1159/000356760

Allen, A. N., Clarke, R., Shipley, M., \& Leon, D. A. (2019). Adiposity in middle and old age and risk of death from dementia: 40-year follow-up of 19,000 men in the Whitehall study. Age and Ageing, 48, 247-253. doi:10.1093/ageing/afy182

Association, A. D. (2018). 2. Classification and diagnosis of diabetes: standards of medical care in diabetes-2018. Diabetes Care, 41(Supplement 1), S13-S27.

Bates, L. M., Acevedo-Garcia, D., Alegría, M., \& Krieger, N. (2008). Immigration and generational trends in body mass index and obesity in the United States: results of the National Latino and Asian American Survey, 2002-2003. American Journal of Public Health, 98, 70-77. doi:10.2105/AJPH.2006.102814

Bell, S. P., Liu, D., Samuels, L. R., Shah, A. S., Gifford, K. A., Hohman, T. J., \& Jefferson, A. L. (2017). Late-life body mass index, rapid weight loss, apolipoprotein $\mathrm{E} \varepsilon 4$ and the risk of cognitive decline and incident dementia. The Journal of Nutrition, Health \& Aging, 21, 1259-1267. doi:10.1007/ s12603-017-0906-3

Carey, R. M., \& Whelton, P. K. (2018). Prevention, detection, evaluation, and management of high blood pressure in adults: Synopsis of the 2017 American College of Cardiology/American Heart Association Hypertension Guideline. Annals of Internal Medicine, 168(5), 351-358. doi: 10.7326/M17-3203

Cetin, D. C., \& Nasr, G. (2014). Obesity in the elderly: more complicated than you think. Cleveland Clinic Journal of Medicine, 81, 51-61. doi:10.3949/ccjm.81a.12165

Cova, I., Clerici, F., Rossi, A., Cucumo, V., Ghiretti, R., Maggiore, L.,...Caracciolo, B. (2016). Weight loss predicts progression of mild cognitive impairment to Alzheimer's disease. PLoS One, 11, e0151710. doi:10.1371/journal.pone.0151710

Cramm, J. M., van Dijk, H. M., \& Nieboer, A. P. (2013). The importance of neighborhood social cohesion and social capital for the well being of older adults in the community. The Gerontologist, 53, 142-152. doi:10.1093/geront/gns052

Diez Roux, A. V., \& Mair, C. (2010). Neighborhoods and health. Annals of the New York Academy of Sciences, 1186, 125-145. doi:10.1111/j.1749-6632.2009.05333.x

Durazo, E. M., Mbassa, R. S., \& Albert, M. A. (2016). Ethnic enclaves and type II diabetes: a focus on Latino/Hispanic Americans. Current Cardiovascular Risk Reports, 10(11), 36. doi:10.1007/s12170-016-0518-1

Echeverría, S., Diez-Roux, A. V., Shea, S., Borrell, L. N., \& Jackson, S. (2008). Associations of neighborhood problems and neighborhood social cohesion with mental health and health behaviors: the Multi-Ethnic Study of Atherosclerosis. Health \& Place, 14, 853-865. doi:10.1016/j.healthplace.2008.01.004

Escarce, J. J., Morales, L. S., \& Rumbaut, R. G. (2006). The health status and health behaviors of Hispanics. In M. Tienda \& F. Mitchell (Eds.), Hispanics and the Future of America (pp. 362409). Washington, DC: National Academies Press.

Eschbach, K., Ostir, G. V., Patel, K. V., Markides, K. S., \& Goodwin, J. S. (2004). Neighborhood context and mortality among older Mexican Americans: is there a barrio advantage? American Journal of Public Health, 94, 1807-1812. doi:10.2105/ajph.94.10.1807 
Fakhouri, T. H., Ogden, C. L., Carroll, M. D., Kit, B. K., \& Flegal, K. M. (2012). Prevalence of obesity among older adults in the United States, 2007-2010. US Department of Health and Human Services, Centers for Disease Control and Prevention, National Center for Health Statistics, Maryland.

Gaddey, H. L., \& Holder, K. (2014). Unintentional weight loss in older adults. American Family Physician, 89, 718-722.

González, H. M., Haan, M. N., \& Hinton, L. (2001). Acculturation and the prevalence of depression in older Mexican Americans: baseline results of the Sacramento Area Latino Study on Aging. Journal of the American Geriatrics Society, 49, 948-953. doi:10.1046/j.1532-5415.2001.49186.x

Grafova, I. B., Freedman, V. A., Kumar, R., \& Rogowski, J. (2008). Neighborhoods and obesity in later life. American Journal of Public Health, 98, 2065-2071. doi:10.2105/AJPH.2007.127712

Haan, M. N., Mungas, D. M., Gonzalez, H. M., Ortiz, T. A., Acharya, A., \& Jagust, W. J. (2003). Prevalence of dementia in older latinos: the influence of type 2 diabetes mellitus, stroke and genetic factors. Journal of the American Geriatrics Society, 51, 169-177. doi:10.1046/j.1532-5415.2003.51054.x

Han, T. S., Tajar, A., \& Lean, M. E. (2011). Obesity and weight management in the elderly. British Medical Bulletin, 97, 169-196. doi:10.1093/bmb/ldr002

Howel, D. (2012). Waist circumference and abdominal obesity among older adults: patterns, prevalence and trends. PLoS One, 7, e48528. doi:10.1371/journal.pone.0048528

HSPH, H. T. H. C. S. o. P. H.-. (2019). The Nutrition Source: Measuring Physical Activity. Retrieved from https://www.hsph. harvard.edu/nutritionsource/mets-activity-table/

Isasi, C. R., Ayala, G. X., Sotres-Alvarez, D., Madanat, H., Penedo, F., Loria, C. M.,...Schneiderman, N. (2015). Is acculturation related to obesity in Hispanic/Latino adults? Results from the Hispanic community health study/study of Latinos. Journal of Obesity, 2015, 186276. doi:10.1155/2015/186276

Ishizawa, H., \& Jones, A. (2016). Immigrant neighborhood concentration, acculturation and obesity among young adults. Journal of Urban Affairs, 38, 298-311. doi:10.1111/juaf.12208

Jackson, H. (2015). Can weight loss explain the obesity-mortality paradox?: key findings from a multi-state model. The Gerontologist, 55(Suppl_2), 464.doi:10.1093/geront/gnv196.08

Jensen, M. D., Ryan, D. H., Apovian, C. M., Ard, J. D., Comuzzie, A. G., Donato, K. A.,...Yanovski, S. Z. (2014). 2013 AHA/ACC/TOS guideline for the management of overweight and obesity in adults. Journal of the American College of Cardiology, 63(25 Part B), 2985. doi: 10.1016/j.jacc.2013.11.004

Jiang, M., Zou, Y., Xin, Q., Cai, Y., Wang, Y., Qin, X., \& Ma, D. (2018). Dose-response relationship between body mass index and risks of all-cause mortality and disability among the elderly: a systematic review and meta-analysis. Clinical Nutrition. doi:10.1016/j.clnu.2018.07.021

Karahalios, A., English, D. R., \& Simpson, J. A. (2016). Change in body size and mortality: a systematic review and meta-analysis. International Journal of Epidemiology, 46(2), 526-546. doi:10.1093/ije/dyw246

Klein, S., Allison, D. B., Heymsfield, S. B., Kelley, D. E., Leibel, R. L., Nonas, C., \& Kahn, R. (2007). Waist circumference and cardiometabolic risk: a consensus statement from shaping america's health: association for weight management and obesity prevention; NAASO, the obesity society; the American society for nutrition; and the American diabetes association. Obesity (Silver Spring, Md.), 15, 1061-1067. doi:10.1038/oby.2007.632

Lavie, C. J., McAuley, P. A., Church, T. S., Milani, R. V., \& Blair, S. N. (2014). Obesity and cardiovascular diseases: implications regarding fitness, fatness, and severity in the obesity paradox. Journal of the American College of Cardiology, 63, 1345-1354. doi:10.1016/j.jacc.2014.01.022

Li, K., Wen, M., \& Henry, K. A. (2017). Ethnic density, immigrant enclaves, and Latino health risks: a propensity score matching approach. Social Science \& Medicine (1982), 189, 44-52. doi:10.1016/j.socscimed.2017.07.019

López, L., Peralta, C. A., Lee, A., Zeki Al Hazzouri, A., \& Haan, M. N. (2014). Impact of acculturation on cardiovascular risk factors among elderly Mexican Americans. Annals of Epidemiology, 24, 714-719. doi:10.1016/j.annepidem.2014.07.011

Markides, K. S., \& Rote, S. (2019). The healthy immigrant effect and aging in the United States and other Western countries. The Gerontologist, 59, 205-214. doi:10.1093/geront/gny136

Mathus-Vliegen, E. M. H. (2012). Obesity and the elderly. Journal of Clinical Gastroenterology, 46(7). doi: 10.1097/ MCG.0b013e31825692ce

McGrath, R., Vincent, B. M., Al Snih, S., Markides, K. S., \& Peterson, M. D. (2017). The association between muscle weakness and incident diabetes in older Mexican Americans. Journal of the American Medical Directors Association, 18, 452.e7-452. e12. doi:10.1016/j.jamda.2017.01.017

Miller, S. L., \& Wolfe, R. R. (2008). The danger of weight loss in the elderly. The Journal of Nutrition, Health o Aging, 12, 487-491. doi:10.1007/bf02982710

Moore, L. V., \& Diez Roux, A. V. (2006). Associations of neighborhood characteristics with the location and type of food stores. American Journal of Public Health, 96, 325-331. doi:10.2105/ AJPH.2004.058040

Murillo, R., Echeverria, S., \& Vasquez, E. (2016). Differences in neighborhood social cohesion and aerobic physical activity by Latino subgroup. SSM - Population Health, 2, 536-541. doi:10.1016/j.ssmph.2016.08.003

Ogden, C. L., Carroll, M. D., Fryar, C. D., \& Flegal, K. M. (2015). Prevalence of obesity among adults and youth: United States, 2011-2014. NCHS Data Brief, 1-8.

Osypuk, T. L., Diez Roux, A. V., Hadley, C., \& Kandula, N. R. (2009). Are immigrant enclaves healthy places to live? The multi-ethnic study of atherosclerosis. Social Science \& Medicine (1982), 69, 110-120. doi:10.1016/j.socscimed.2009.04.010

Park, S. Y., Wilkens, L. R., Maskarinec, G., Haiman, C. A., Kolonel, L. N., \& Marchand, L. L. (2018). Weight change in older adults and mortality: the Multiethnic Cohort Study. International Journal of Obesity (2005), 42, 205-212. doi:10.1038/ijo.2017.188

Pérez, D. J., Fortuna, L., \& Alegria, M. (2008). Prevalence and correlates of everyday discrimination among U.S. latinos. Journal of Community Psychology, 36, 421-433. doi:10.1002/ jcop. 20221

Portes, A., \& Manning, R. D. (1986). The immigrant enclave: Theory and empirical examples 47-68.

Rodriguez, F., Hu, J., Kershaw, K., Hastings, K. G., López, L., Cullen, M. R.,...Palaniappan, L. P. (2018). County-level hispanic 
ethnic density and cardiovascular disease mortality. Journal of the American Heart Association, 7, e009107. doi:10.1161/ JAHA.118.009107

Rothman, K. J. (2008). BMI-related errors in the measurement of obesity. International Journal of Obesity (2005), 32 Suppl 3, S56-S59. doi:10.1038/ijo.2008.87

Schisterman, E. F., Cole, S. R., \& Platt, R. W. (2009). Overadjustment bias and unnecessary adjustment in epidemiologic studies. Epidemiology (Cambridge, Mass.), 20, 488-495. doi:10.1097/ EDE.0b013e3181a819a1

Shea, M. K., Nicklas, B. J., Houston, D. K., Miller, M. E., Davis, C. C., Kitzman, D. W.,...Kritchevsky, S. B. (2011). The effect of intentional weight loss on all-cause mortality in older adults: results of a randomized controlled weight-loss trial. The American Journal of Clinical Nutrition, 94, 839-846. doi:10.3945/ajen.110.006379

Turra, C. M., \& Elo, I. T. (2008). The impact of salmon bias on the hispanic mortality advantage: new evidence from social security data. Population Research and Policy Review, 27, 515-530. doi:10.1007/s11113-008-9087-4

Ullmann, S. H., Goldman, N., \& Pebley, A. R. (2013). Contextual factors and weight change over time: a comparison between U.S. Hispanics and other population sub-groups. Social Science \& Medicine (1982), 90, 40-48. doi:10.1016/j. socscimed.2013.04.024

Viruell-Fuentes, E. A., Morenoff, J. D., Williams, D. R., \& House, J. S. (2013). Contextualizing nativity status, Latino social ties, and ethnic enclaves: an examination of the 'immigrant social ties hypothesis'. Ethnicity or Health, 18, 586-609. doi:10 $.1080 / 13557858.2013 .814763$

Wang, Y. C., Colditz, G. A., \& Kuntz, K. M. (2007). Forecasting the obesity epidemic in the aging U.S. population. Obesity (Silver Spring, Md.), 15, 2855-2865. doi:10.1038/oby.2007.339
Whitley, J. C., Peralta, C. A., Haan, M., Aiello, A. E., Lee, A., Ward, J.,...López, L. (2018). The association of parental and offspring educational attainment with systolic blood pressure, fasting blood glucose and waist circumference in Latino adults. Obesity Science \& Practice, 4, 582-590. doi:10.1002/ osp4.307

Whitmer, R. A., Gunderson, E. P., Barrett-Connor, E., Quesenberry, C. P. Jr, \& Yaffe, K. (2005). Obesity in middle age and future risk of dementia: a 27 year longitudinal population based study. BMJ (Clinical Research ed.), 330, 1360. doi:10.1136/bmj.38446.466238.E0

Whitmer, R. A., Gustafson, D. R., Barrett-Connor, E., Haan, M. N., Gunderson, E. P., \& Yaffe, K. (2008). Central obesity and increased risk of dementia more than three decades later. Neurology, 71, 1057-1064. doi:10.1212/01.wnl.0000306313.89165.ef

Witham, M. D., \& Avenell, A. (2010). Interventions to achieve long-term weight loss in obese older people: a systematic review and meta-analysis. Age and Ageing, 39, 176-184. doi:10.1093/ ageing/afp251

Yen, I. H., Michael, Y. L., \& Perdue, L. (2009). Neighborhood environment in studies of health of older adults: a systematic review. American Journal of Preventive Medicine, 37, 455-463. doi:10.1016/j.amepre.2009.06.022

Zajacova, A., \& Ailshire, J. (2014). Body mass trajectories and mortality among older adults: a joint growth mixture-discrete-time survival analysis. The Gerontologist, 54, 221-231. doi:10.1093/ geront/gns164

Zeki Al Hazzouri, A., Haan, M. N., Osypuk, T., Abdou, C., Hinton, L., \& Aiello, A. E. (2011). Neighborhood socioeconomic context and cognitive decline among older Mexican Americans: results from the sacramento area latino study on aging. American Journal of Epidemiology, 174(4), 423-431. doi:10.1093/aje/kwr095 